\title{
Carnets
}

Revue électronique d'études françaises de l'APEF

Première Série - 5 | 2013

Métamorphoses littéraires

\section{La microfiction comme métamorphose du conte: Éclatement narratif et transfictionnalité dans Petits Chaperons de José Luis Zárate}

\section{Cristina Álvares}

\section{(2) OpenEdition \\ Journals}

Édition électronique

URL : http://journals.openedition.org/carnets/8382

DOI : $10.4000 /$ carnets.8382

ISSN : 1646-7698

Éditeur

APEF

Édition imprimée

Date de publication : 1 mai 2013

Pagination : 143-163

Référence électronique

Cristina Álvares, « La microfiction comme métamorphose du conte: Éclatement narratif et

transfictionnalité dans Petits Chaperons de José Luis Zárate », Carnets [En ligne], Première Série -

5 | 2013, mis en ligne le 23 juin 2018, consulté le 01 mai 2019. URL : http://journals.openedition.org/ carnets/8382 ; DOI : 10.4000/carnets.8382

\section{(9) $(1) \Theta$}

Carnets est mis à disposition selon les termes de la licence Creative Commons - Atribution - Pas d'utilisation commerciale 4.0 International. 


\section{LA MICROFICTION COMME MÉTAMORPHOSE DU CONTE Éclatement narratif et transfictionnalité dans Petits Chaperons de José Luis Zárate ${ }^{1}$}

CRISTINA Álvares

Universidade do Minho calvares@ilch.uminho.pt

\section{Résumé}

Pour situer Petits Chaperons de José Luis Zárate dans le débat autour du rapport génologique, voire généalogique, de la microfiction au conte, l'article présente et discute l'œuvre à trois niveaux. Premièrement, celui du statut interlinguistique et intermédial qui préside à sa production et publication. Deuxièmement, sur le plan de la forme narrative et intertextuelle, on examine l'impact de la série microfictionnelle sur la syntagmatique narrative et l'unité diégétique du conte, en particulier les métamorphoses subies par le temps au sein de chaque micronouvelle (comprimé en un "récit sans narrativité") ainsi que dans leur rapport les unes aux autres (absence de chrono-logie). Troisièmement, nous analysons les figures d'un lien intertextuel spécifique, appelé transfictionnalité, situé au niveau du contenu diégétique et thématique, où la connexion des microfictions au conte s'établit par partage d'éléments fictifs.

\section{Abstract}

In order to locate Petits Chaperons de José Luis Zárate in the debate about the genre relations between microfiction and short story, this paper features and discusses Zárate's work at three levels. Firstly, the interlinguistic and intermedial status that presides over its production and publishing. Secondly, we examine on the intertextual field the impact of the microfictional series onto the narrative syntagmatics and the diegetic unity of the short story, particularly changes undertaken by time within every microfiction (compressed into a "narrative without narrativity") and within the overall set as well (no chrono-logics). Thirdly, we look into the operations of a specific intertextual link, called transfictionality, located in the diegetic contents where the connection between microfiction and short story is studied within the frame of the fictional and thematic unity of the microfictional series.

Mots-clés: conte, microfiction, série, transfictionnalité

Key-words: short story, microfiction, series, transfictionality

\footnotetext{
${ }^{1}$ Cet article a été produit dans le cadre du projet de recherche PTDC/CLE-LLI/103972/2008 Mutações do conto nas sociedades urbanas contemporâneas, financé par la Fundação para a Ciência e Tecnologia.

Cristina Álvares, "La microfiction comme métamorphose du conte: Éclatement narratif et transfictionnalité dans Petits Chaperons de José Luis Zárate”, Carnets V, Metamorphoses Litteráires, mai 2013, pp. 143-163 


\section{Conte et microfiction}

Que la microfiction, forme qui cherche l'expression minimale du narratif, soit une mutation du conte est une idée assez répandue et consensuelle. En contexte hispanoaméricain, où la production et l'étude de cette modalité d'écriture se sont le plus développées, les formes et régimes de cette mutation font l'objet d'un débat chez les critiques et les théoriciens hispanophones. En témoigne le recueil d'articles organisé par David Roas sous le titre de Poéticas del microrrelato, publié en 2010. Directement associée au problème de l'autonomie du genre, posée par certains théoriciens, comme David Lagmanovitch et Lauro Zavala, niée par d'autres, comme David Roas, la question de la genèse du microrrelato ou micro- ou minicuento est placée en référence au conte. Aussi, Roas affirme-t-il que le microcuento ou minicuento est une variante ou forme radicale et expérimentale du conte littéraire moderne, tel que Edgar Allan Poe l'a théorisé ${ }^{2}$ : son trait majeur, the single effect, résulte de la brièveté et de la cohérence d'un récit made to the point pour être lu at one sitting. D'autre part, David Lagmanovitch tout comme Irene AndresSuárez reconnaissent que le microrrelato dérive effectivement du conte mais il pensent au contraire que la recherche de la concision et de l'intensité narratives subit à un moment donné une transition brusque signalant une mutation structurale qui élève la forme narrative brève et hyperbrève à la condition de genre autonome. Une troisième position existe qui, tout en soulignant la transgénéricité et donc le statut génologiquement dépendant du microrrelato, met en doute que le genre tutélaire soit le seul conte. Dans le courant transgénérique, Zavala, tout en mettant en relief la nature génologiquement hybride et frontalière de la microfiction, la considère un genre et l'étudie en connexion privilégiée avec le conte ${ }^{3}$.

Le lien de dérivation, continu ou discontinu, de la microfiction au conte trouve également une expression en portugais - microconto - et en anglais - short short story. Ces désignations coexistent dans les deux langues avec d'autres comme flash fiction, sudden fiction, microficção ou micronarrativa ${ }^{4}$. Mais en français il n'y a pas de désignation équivalente à microcuento. L'expression "contes ultra-brefs" qualifie les contes de Jacques Sternberg. Les désignations les plus utilisées sont "microfiction" - titre d'un recueil de récits

\footnotetext{
2 II va sans dire que théorie et pratique ne coïncident pas toujours et qu'il est facile de trouver des contes excentriques par rapport au modèle de Poe (cf. Lawrence, 1917).

${ }^{3}$ C'est bien ce qu'indiquent les titres de ses ouvrages et articles - par exemple, "La teoría del cuento y la minificción en Venezuela" -, ainsi que son site El cuente en red. Revista eletrónica de teoría de la ficción breve, où l'on trouve des études sur le conte et la microfiction.

${ }^{4}$ Le terme microficción ou minificción existe également en espagnol. Lauro Zavala distingue minicuento et minificción en tant que catégories du microrrelato. Le premier se concentre sur l'histoire dont il garde l'intégrité et le temps séquentiel (final anaphorique); la seconde décentre ou disloque l'histoire, dont l'intégrité est affectée, l'ordre du temps étant rétrospectif avec final cataphorique (Zavala, s/d).
} 
brefs de Régis Jauffret - et "micronouvelle" qui est le terme retenu par des auteurs plus périphériques que Jauffret comme Jacques Fuentealba, Vincent Bastin et le groupe québécois Oxymorons. Le terme "microrécit" apparaît quelques fois. II y a aussi des récits qui présentent les caractéristiques fondamentales de la micronouvelle sans en porter le nom. Entre autres: les nouvelles en trois lignes de Jean-Louis Bailly, ou en quatre lignes de JeanNoël Blanc, deux auteurs qui ont tout récemment repris la forme inventée par Félix Fénéon il y a cent ans; les nouvelles de Chantal Thomas dont un certain nombre au moins tombe sous la coupe structurale de la micronouvelle; les posts quotidiens d'Éric Chévillard sur son blog L'autofictif; les expériences numériques de François Bon (Bonnet, 2011).

Les deux termes les plus utilisés en français, "micronouvelle" et "microfiction" indiquent que le monde littéraire francophone dissocie le récit bref ou ultrabref et le conte. Si "microfiction" signifie la nature transversale de la fiction aux genres, aux medias et aux arts qui supportent matériellement ces récits, "micronouvelle', de son côté, spécifie leur lien au genre littéraire de la nouvelle. Je ne souhaite pas m'attarder à spéculer sur d'éventuelles implications esthétiques et culturelles à tirer de cette différence terminologique. II suffit de remarquer que la nouvelle partage avec le conte l'unité et l'intensité de l'effet (la tension vers une chute, l'impact du final unique sur la saisie de l'instant (cf. Bonnet, 2011: 9). Dans Notes Nouvelles sur Edgar Poe (1859), Baudelaire attribue à la nouvelle le trait majeur que Poe attribue au conte: "la nouvelle a sur le roman à vastes proportions cet immense avantage que sa brièveté ajoute à l'intensité de l'effet" (Baudelaire, 1965: 35). La nouvelle est le genre qui inscrit la fiction dans la fugacité du temps. La brièveté plus resserrée de la micronouvelle découle de l'expression maximale des potentialités structurales communes à ces deux genres narratifs brefs, le conte et la nouvelle. Ce disant, je ne prétends pas gommer l'écart conceptuel, fût-il minuscule, qu'il y a entre microcuento et "micronouvelle" et qui signale une nuance dans la perception du phénomène en contexte francophone. L'option pour la nouvelle assume tacitement ses conventions spécifiques: les événements racontés se seraient réellement et récemment produits, la syntagmatique narrative est moins consistante que dans le conte, la signification est plus dépendante du contexte.

\section{Contes et contes de fées}

En affirmant son lien intertextuel à un conte particulier, Petits Chaperons de José Luis Zárate se place au sein de ce débat. L'œuvre présente un double statut linguistique et médial que l'on résumera de la façon suivante: twittérature en espagnol, littérature en français. Un auteur mexicain poste en espagnol et publie en français des récits minuscules ne dépassant pas les 140 caractères. Son blog parle de tuiteratura et de mini et microcuentos, mais la quatrième de couverture de Petits Chaperons parle de micronouvelles 
et nous dit que Zárate, "chef de file de la SF et du fantastique, publie sur Twitter des séries de micronouvelles autour de contes ou de personnages célèbres, tels que Shéhérazade, Icare ou Hyde". La phrase définit les micronouvelles de Zárate comme des réécritures de contes. D'après les personnages nommés, il semble qu'il s'agit de fictions dont la prégnance imaginaire leur donne une place spéciale dans notre mémoire collective, si bien qu'elles mènent une existence en quelque sorte autonome par rapport aux œuvres littéraires qui les ont créées et que leurs personnages ont un statut assez proche de celui de l'icône culturel. Petits Chaperons reprend une des ces fictions célèbres dont la forme narrative et génologique est celle du conte, plus précisément le conte de fées. Ce faisant, Petits Chaperons prend place dans le débat autour du rapport entre micronouvelle et conte, mais cette place est en quelque sorte déplacée ou en marge de la question centrale car il ne va pas de soi que le conte auquel les micronouvelles sont liées soit un conte littéraire moderne.

Écrits par Perrault dans le milieu aristocratique français de la fin du XVIle siècle, les Contes ont subi plusieurs reconfigurations et sédimentations, dont les plus marquantes sont sans doute celles des frères Grimm au XIXe siècle et de Disney au XXe siècle, qui les adaptent à de nouveaux contextes socioculturels et technologiques et à de nouvelles attentes esthétiques et idéologiques (bourgeoisie protestante, romantisme allemand, culture de masses). À la suite des rééditions des Contes par le Cabinet des fées au long du XVIIIle siècle, les frères Grimm (et plus tard Disney) ont établi le canon des "contes de fées" conçus comme des récits archaïques d'origine populaire destinés aux enfants ${ }^{5}$. Cette perception des contes, filtrée par une fiction romantique mise en place par Grimm (Belmont, 1986, 1999), a traversé tout le XXe siècle, légitimée par l'anthropologie et la psychanalyse qui lui apportent une sanction scientifique ${ }^{6}$. Walter Benjamin en est un représentant. On dirait, dans ses termes, que le fairy tale rentre dans la catégorie du tale, lequel, disait Walter Benjamin, n'est pas la même chose que le short story, parce que le tale est le genre clé de la "narration artisanale', tandis que le short story est un genre de l'époque de la reproduction mécanisée

\footnotetext{
${ }^{5}$ L'édition de 1695 de Contes chez Barbin en est responsable à travers le péritexte formé par le titre et le frontispice. Le titre Contes de ma mere loye est illustré par le frontispice montrant une vieille nourrice qui, tout en filant, raconte des histoires à un petit groupe d'enfants qui écoutent attentivement. Au-dessus une plaque répète le titre. L'expression "contes de ma mère l'oie" signifie, selon le Dictionnaire de l'Académie Française de 1694, "des fables ridicules telles que sont celles dont les vieilles gens entretiennent et amusent les enfants" (apud Heidmann et Adam, 2010:201). En lisant Heidmann et Belmont, on peut penser que le péritexte est une stratégie éditoriale visant à investir le livre de ce que Benjamin appelle l'aura - "l'apparition unique d'un lointain, si proche soit-il" (Benjamin, 1991:144). Pour la conception enfantine des contes chez Grimm (Kinder Marchen), voir Belmont, 1999.

${ }^{6}$ L'origine orale et populaire a été en général reçue comme une évidence mais la vocation enfantine des contes ne fait pas l'unanimité.Certains auteurs supposent que dans leur forme orale originaire, les contes se destinaient aux adultes et que c'est leur mise en texte qui en a fait de la littérature infantile. Clarissa Pinkola Estés regrette la dépaganisation des contes opérée par les frères Grimm et la désexualisation qui en résulte. "La plupart des anciens recueils de contes de fées et les récits mythologiques qui sont parvenus jusqu'à nous ont perdu en route leurs éléments scatologiques, sexuels, pervers (par le biais des mises en garde), féminins, initiatiques, préchrétiens" (Pinkola Estés, 1996:33). Nicole Belmont pense également que l'écriture a dénaturé les contes (cf. Belmont, 1999).
} 
des textes (Benjamin,1991:214) ${ }^{7}$. La formule "il était une fois" les pourvoit d'une aura qui les renvoie au temps jadis. Mais des études récentes comme celles de Ute Heidmann et JeanMichel Adam ont vigoureusement ébranlé la thèse tenace de l'origine populaire et orale des contes ainsi que de leur destinataire enfantin exclusif ou prioritaire (Heidmann et Adam, 2010; Belmont, 1999). Heidmann et Adam soutiennent l'origine littéraire des Contes de Perrault, qui s'élaborent dans un dialogue intertextuel à maille serrée avec les contes et les nouvelles d'Apulée, Boccace, Basile, Straparola, Aulnoy, Lhéritier ${ }^{8}$. L'analyse philologique et linguistique des contes et de leurs réécritures et reconfigurations depuis Perrault oblitère l'idée romantique d'une convergence entre spontanéité du peuple et naïveté des enfants. Bien enracinée dans les sciences humaines (anthropologie, ethnologie, études littéraires, psychanalyse) ainsi que dans le sens commun, l'illusion archaïque concernant les contes de fées guide une tradition herméneutique qui les constitue comme une catégorie à part dans le champ générique du conte: ce ne sont pas des contes littéraires modernes, ce sont de très vieux contes pour enfants. Pourtant, les contes de fées et notamment le Petit Chaperon Rouge, le plus court des contes de Perrault, confirment la caractéristique sine qua non du conte selon Poe: l'unité d'effet. Du point de vue de la structure et de la forme narrative, les contes de fées sont des contes. C'est plutôt au niveau du contenu diégétique et de la morphologie des mondes de fiction que se situent les propriétés particulières qui les constituent comme une variante ou un sous-genre du conte. Leur reprise et circulation constante dans le paysage culturel créé des dynamiques d'intersection entre les champs littéraire et médiatique qui montrent qu'ils jouissent d'une longévité et d'une vitalité méconnues de la plupart des autres contes ${ }^{9}$.

\section{Petits Chaperons: de la twittérature à l'œuvre littéraire}

Les micronouvelles que Zárate a dédiées au Petit Chaperon Rouge ont été traduites de l'espagnol en français par Jacques Fuentealba, lui-même auteur et critique de micronouvelles, spécialiste des genres populaires et traducteur d'auteurs hispaniques. Petits

\footnotetext{
${ }^{7}$ Benjamin a écrit Le narrateur en 1936, soit une année avant la sortie dans les cinémas américains de Snow White and the Seven Dwarfs par les studios Disney.

${ }^{8}$ Le Petit Chaperon Rouge s'élabore comme une inversion symétrique du conte d'Apulée Psyché (Heidmann et Adam, 2010:81-5).

${ }^{9}$ La place à part des contes de fées au sein du genre conte est corrélative de leur disponibilité transfictionnelle. Ils constituent une région spéciale de notre imaginaire et leurs fictions se comptent entre les plus reprises et recyclées par des textes, des médias et des arts différents, dans une interaction vigoureuse entre culture littéraire et culture populaire et médiatique. Il semble que le recyclage des contes suive deux lignes d'orientation: l'enchantée (ou édulcorée), qui reconfigure les histoires dans un sens rassurant pour les enfants (c'est la voie de Grimm et de Disney); la désenchantée (ou déniaisée), sous les registres dérisoire et comique (les dessins animés de Tex Avery, le cycle Shrek) ou sombre, cynique ou tragique: Chapéuzinho Vermelho, de Donald Trevisan (Trevisan, 2003:72-4), les récentes dark fantasies cinématographiques dédiées au Petit Chaperon et à Blancheneige ou la série de photographies Fallen Princesses de Dina Goldstein (2009). Destinées à tous publics ou prioritairement aux adultes, les versions désenchantées soustraient les fées aux contes.
} 
Chaperons a été publié en mini-livre $(7 \mathrm{~cm} / 9 \mathrm{~cm})$, dans une collection consacrée à la microfiction dirigée par Fuentealba, chez Outworld ${ }^{10}$. On remarquera la double transposition subie par les micronouvelles de Zárate: traduites de l'espagnol en français, elles se sont déplacées de Twitter au livre imprimé. Ces textes sont des hybrides linguistiques et médiaux. La transposition des textes d'un espace online à un espace offline entraîne un régime de lecture différent, car on ne lit pas une série numérique comme on lit un recueil imprimé. Le mini-livre est une série finie, un objet qui résulte du découpage d'un segment prélevé sur le flux numérique virtuellement infini et qui a une forme déterminée, une œuvre. Ce segment (le recueil) assume dans le livre une forme linéaire inchangeable qui propose un ordre de lecture. Au lecteur de le suivre ou non, car Petits Chaperons peut se lire dans n'importe quel ordre. Sur Twitter les textes se succèdent verticalement, en liste, tout en étant susceptibles de lectures multilinéaires, réticulaires et interactives, ce qui est impossible dans le livre. Comme Dominique Faria l'a montré en parlant du blog de Chévillard, Internet permet au lecteur de bâtir de multiples parcours de lecture dont l'interactivité établit entre lui et l'auteur un lien de proximité qui se trouve annulé dans le livre (Faria, 2009). Aussi la double transposition interlinguistique et intermédiale des micronouvelles de Zárate, opérée par Fuentealba, accomplit-elle la transition entre la twittérature ${ }^{11}$, autrement dit le numérique, le continu, le processus, l'éphémère, l'accès gratuit, et l'œuvre littéraire avec ses effets et attributs: le livre, le discontinu, l'achevé, le durable, la valeur marchande. Cette position signale la double appartenance des micronouvelles aux espaces para-institutionnels et aux circuits culturels officiels.

\section{Récit sans narrativité, narrativité sans récit}

Écrits par Fuentealba, les éléments péritextuels de l'œuvre rattachent les micronouvelles aux contes. La quatrième de couverture présente les contes comme leur modèle et référence et le titre explicite le lien intertextuel de ces micronouvelles à un conte particulier. Petits Chaperons indique que nous avons affaire à une réécriture du Petit Chaperon Rouge orientée et marquée par la pluralisation. Le projet n'est pas nouveau. En 1989, Gilbert Lascault avait publié Le Petit Chaperon Rouge, partout, série de cinquante textes courts et très courts, lesquels évidemment n'ont pas été préalablement postés sur Twitter. Dans les récits de Lascault le personnage principal est doté d'ubiquité internationale et transhistorique: il est dans le Titanic, apparaît en eskimo, déclenche la révolution d'octobre

\footnotetext{
10 Outworld est un éditeur marginal, voire contreculturel, dont le nom signifie sa position ex-centrique (horsmonde) tandis que le recours à l'anglais souligne sa visée de communication globale (dans le monde). http://www.outworldeditions.com/

${ }_{11}$ L'Institut de Twittérature Comparée (ITC) a été fondé à Montréal et à Bordeaux en 2010 par Jean-Yves Fréchette et Jean-Michel Le Blanc http://www.twittexte.com/ScriptorAdmin/scripto.asp?resultat=734326.
} 
à Saint Petersbourg, rencontre la Belle au Bois Dormant. Le conte est réécrit en récit biblique de la création, en dissertation universitaire, reconfiguré en jeu de cartes. Chez Zárate, la dispersion du personnage et du conte est d'une autre sorte.

Les micronouvelles reprennent un récit tellement populaire qu'il n'est même pas représenté par un texte particulier, celui de Perrault ou celui de Grimm, mais par une version de référence ou officielle ou standard qui mélange des éléments des deux textes. Bien que systématiquement référée à Perrault, la fiction de Zárate fait intervenir le Chasseur, personnage créé par Grimm pour donner un dénouement heureux au conte ${ }^{12}$. Les micronouvelles ne sont donc pas lisibles en tant que textes indépendants. Leur intelligibilité dépend de la mémoire du conte qui doit être rappelé et réactivé pour que la signification parodique et humoristique de chaque micronouvelle soit pleinement appréciée. Un texte aussi dépouillé comme "Le GPS du Petit Chaperon gâcha le conte" (78) n'aura pas de sens pour un lecteur méconnaissant Le Petit Chaperon Rouge. La lecture des unes exige la réactivation de l'autre.

Chacune des micronouvelles est un récit autonome qui donne une version parodique et humoristique du conte, réduit à un détail, à une action ou à un instant: "Avec quelle joie le Petit Chaperon rouge se précipite dans le bois avec le goûter destiné à sa grand-mère depuis longtemps morte.'(4) ${ }^{13}$; "Les bêtes attaquent en meute, elles tuent en groupe. L'un traita le Petit Chaperon de catin, l'autre la frappa, le village entier marcha sur elle'.(57); Le jeune homme regarda son duvet pubien, sûr que la transformation qui comblerait le Petit Chaperon rouge venait de commencer (22); "Le chasseur sortit du loup, miraculeusement encore en vie, le Petit Chaperon, la mère-grand, trois petits cochons et un homme perdu qui dit s'appeler Geppetto'.(23). Dans chaque micronouvelle un segment de l'histoire est donné en bloc, d'une seule fois, dans un récit singulatif qui emploie soit le passé simple (traita, frappa, marcha, regarda, sortit), soit le présent historique (se précipite). L'ellipse condense et comprime à l'extrême la syntagmatique narrative interne à chaque récit, si bien que le temps ne se déroule pas en temporalité mais se replie et concentre sur l'instant de l'action isolée et, quelques fois, de son résultat immédiat. Dans celle-ci, le cadavre du Petit Chaperon est le résultat direct du vol de la hache: "La hache vola. Le village entier accueillit le chasseur en héros quand il revint avec le cadavre de cette dévergondée de Petit Chaperon" (17). Cette contraction de la syntagmatique fait des micronouvelles des "récits sans narrativité" (Macé, 2010: 218).

\footnotetext{
${ }^{12}$ Par contre on y retrouve la mère insoucieuse de Perrault et que Grimm remplace par la mère qui avertit sa fille contre les loups. Elle n'apparaît qu'une fois en marâtre, sa fausse insouciance dénoncée comme stratégie meurtrière: "Elle l'envoya vêtue de rouge dans un bois infesté de loups, sans armes ni compagnie. Ils la dévorèrent, mais pas exactement comme le voulait sa mère." (8)

${ }^{13}$ Les pages n'étant pas numérotés, nous indiquons ainsi la position du texte cité dans la série.
} 
À la lecture de cette petite sélection, nous constatons que l'unité et l'autonomie narrative de chaque micronouvelle sont soulignées non seulement par l'action unique, mais aussi par l'absence de continuité logico-narrative entre elles. Le premier récit cité semble répéter le début du conte, lorsque le Petit Chaperon s'apprête à rendre visite à Grand-Mère, mais l'addition du complément temporel "depuis longtemps morte" le relance dans un temps qui s'étend bien après le dénouement. La répétition du début de l'histoire devient alors si ostensiblement absurde - à quoi bon apporter le goûter à quelqu'un qui est déjà mort? - que la joie du personnage prend une signification autre: ce n'est sûrement pas le rendez-vous avec la Grand-Mère qui l'excite mais le rendez-vous avec le Grand Méchant Loup: la tension narrative du conte est remplacée par la tension pulsionnelle dans la micronouvelle. L'innocence du Petit Chaperon Rouge disparaît aussitôt. Le second récit cité présente un dénouement alternatif: le lynchage du Petit Chaperon, dénouement par ailleurs contrarié dans la 17 e micronouvelle où c'est le Chasseur qui tue le Petit Chaperon. Le troisième réécrit le conte dans le registre érotique fantastique (loup-garou). Le quatrième (23) reprend la séquence finale rajoutée par Grimm dans laquelle le Loup accouche du Petit Chaperon et de sa grand-mère, mais aussi de personnages d'autres contes, en l'occurrence les trois petits cochons et le père de Pinocchio.

Cet échantillon montre que les micronouvelles s'organisent selon le principe de la parataxe: elles se succèdent sans ordre, sans hiérarchie et sans cohérence et c'est pourquoi on peut lire le livre dans n'importe quel ordre. La pluralité des Petits Chaperons découle de cette rupture de la syntagmatique narrative du conte en des récits autonomes, brusques et discontinus. La série parataxique opère ainsi la désintégration du conte dans lequel l'histoire se construit progressivement en suivant un enchaînement logique de causalités. II n'y a de déroulement ni internement ni extérieurement dans le rapport des micronouvelles les unes aux autres. Si chaque micronouvelle est en elle-même un tout petit récit, au niveau de la série elles ne forment pas de totalité ou d'unité. II n" y a ni internement ni extérieurement d'enchaînement d'actions à effet de séquence. Internement, la fréquence singulative épuise la temporalité. Extérieurement, aucun ordre temporel ne les lie les unes aux autres. L'unité du conte explose dans le désordre parataxique des petits récits flottants. La série microfictionnelle disperse les actions dans un temps désorienté, ce qui rend impossible au lecteur de reconstituer virtuellement une séquence de l'histoire. La coexistence de plusieurs dénouements incompatibles annule la vectorialité du récit (son mouvement vers l'avant) et rend impossible le single effect. Autrement dit, la série, c'est de la narrativité sans récit. Si l'on compare le conte à un corps, les micronouvelles correspondent à des membres et des organes épars mais aussi à des excroissances et des prothèses (les éléments étrangers au conte) qui dé- et re-composent un montage instable et discontinu évoquant le corps démembré des pulsions, tel que Freud l'a conçu, ou la représentation rabelaisienne du corps 
telle que Bakhtine l'a décrite. C'est donc au niveau de la série, là où la discontinuité est tangible, que chaque micronouvelle, pourtant pourvue d'autonomie lorsque considérée isolément, apparaît comme le fragment d'un tout, en l'occurrence le petit tout qu'est le conte - ou plutôt qu'était le conte, car la série l'a troué et il n'en reste que des bribes qui sont autant de versions partielles, désarticulées et hétéroclites.

II s'établit ainsi une dialectique entre le tout petit (les micronouvelles) et le petit tout (le conte). Ce jeu de mots est emprunté à Pascal Quignard réfléchissant sur la forme fragmentaire en littérature. Le petit tout est en quelque sorte un faux fragment car il est concentré, nucléaire, circulaire, essentiel. L'aphorisme et autres genres gnomiques en sont des exemples. On peine à y découvrir "le pluriel, le mortel, le rompu et le discontinu" (Quignard, 2005: 44). Le fragment qui porte ces traits de pluralité, mortalité, rupture et discontinuité est quelque chose de cassé et de cassant, "pure attaque de prose intense', supposant un flux et non une totalité (idem: 56-70). Dans des ouvrages postérieurs, Quignard appellera ce fragmentaire le sordes. Le jeu de mots petit tout, tout petit se prête à synthétiser le rapport entre le conte et la micronouvelle. Car le conte (contes modernes et prémodernes confondus) est bien le modèle du récit bref pourvu d'unité, de cohérence et de continuité narratives et diégétiques: c'est un petit tout, un petit ordre; tandis que la série de récits hyperbrefs fait éclater cette intégrité narrative en de multiples récits discontinus et logiquement incompatibles: c'est des tout petits, des restes. Dans le conte, le petit garde l'ordre de succession des actions dans une totalité (la clôture du conte); alors que dans les micronouvelles la totalité est absente et la succession ne suit aucun ordre chrono-logique. Le tout petit n'est pas un fragment ou fraction d'une totalité perdue susceptible d'être recomposée: impossible de refaire ou parfaire un conte (une unité narrative) à partir des micronouvelles de Zárate. Les éclats ne collent pas les uns aux autres, les pièces du puzzle ne rentrent pas les unes dans les autres. Leur ensemble à jamais délié, détotalisé, pluriel et ouvert fait obstacle à tout projet d'ordre narratif. L'effet de la série n'est pas de séquence et d'enchaînement, mais d'accumulation parataxique de tout petits récits. C'est de cette façon que la série, tout en déconstruisant le conte, réactive et garde sa mémoire, dans une tension parodique entre dérision et hommage.

La série microfictionnelle se caractérise par ce que Lauro Zavala appelle le fractal, propriété majeure, selon lui, de l'esthétique postmoderne ${ }^{14}$. La micronouvelle apparaît ainsi

\footnotetext{
14 "La serialidad, como ha sido señalado en diversas ocasiones, pone en crisis el concepto moderno de fragmento (y su lugar de origen, es decir, la totalidad), sustituyéndolo por la noción posmoderna de detalle (es decir, de la posibilidad de que este mismo fragmento sea leído de manera totalmente autónoma en relación con la totalidad de la que surge). En el contexto de la teoría literaria, la posibilidad de que un segmento cualquiera de una obra unitaria pueda ser leído indistintamente como fragmento (es decir, como un texto que requiere de la totalidad a la que pertenece para cobrar sentido) o como detalle (es decir, como un texto que puede ser leído de manera completamente independiente de esa totalidad originaria) recibe el nombre de fractal. Un fractal literario
} 
comme le genre littéraire qui radicalise le processus d'érosion que l'expérimentalisme littéraire a fait subir à l'intégrité narrative depuis l'avènement du modernisme et des avantgardes. De ces expériences du début du XXe siècle découle l'esthétique de la brièveté (Andres-Suárez, 2010:36-9, 121; Valls, 2008:30), revivifiée par la déconstruction postmoderne des "grands récits" (Lyotard, 1979) et par les contraintes matérielles et technologiques des nouveaux medias qui ont disponibilisé de nouveaux espaces pour l'écriture et la lecture. Le fait que l'intégrité narrative est ici représentée par un conte conventionnellement classé comme conte de fées et non pas par un conte littéraire moderne a un effet de décentrement ironique par rapport au champ littéraire et à son régime de fonctionnement, ce qui ne semble pas inattendu dans un auteur publiant chez un éditeur périphérique et paralittéraire comme Outworld.

\section{Transfictionnalité}

Et pourtant les micronouvelles de Zárate présentent une unité remarquable assurée par une constante reprise des personnages du Petit Chaperon, du Loup, de la Grand-Mère et du Chasseur. Leur présence est un facteur de cohésion de la série. Grâce à eux nous reconnaissons immédiatement l'histoire que les micronouvelles pulvérisent. Nous entrons dans un monde connu et familier. Si l'organisation parataxique des micronouvelles porte dommage à la syntagmatique narrative, donc au déroulement logique et chronologique de l'histoire, elle n'atteint pourtant pas la stabilité de ce que la théorie de la fiction appelle un univers ou monde fictionnel (Pavel, 1988). Malgré les altérations subies, le monde fictionnel du Petit Chaperon Rouge garde dans Petits Chaperons son identité, grâce surtout à la récurrence des personnages mais aussi des lieux (forêt, village, maison de la Grand-Mère) et des événements majeurs (rencontre avec le Loup, déguisements du Loup, intervention du chasseur en obstétricien). Notre lecture se déplace ainsi du niveau textuel et intertextuel vers le niveau de la fiction dans lequel micronouvelles et conte entretiennent un rapport transfictionnel. Selon Saint-Gélais, qui l'a inventé, le concept de transfictionnalité désigne "le phénomène par lequel au moins deux textes, du même auteur ou non, se rapportent conjointement à la même fiction, que ce soit par reprise de personnages, prolongement d'une intrigue préalable ou partage d'univers fictionnels" (Saint-Gélais, 2011: 7). La transfictionnalité est un cas particulier d'intertextualité qui opère selon une économie et des mécanismes propres.

es simultáneamente un fragmento y un detalle, es decir, es parte de una totalidad (como la novela) y es simultáneamente un texto autónomo (como el que se puede encontrar en una antología)”.(Zavala, 2009: p.41). 
II y a transfictionnalité lorsque deux textes ou davantage "partagent" des éléments fictifs (c'est-à-dire, y font conjointement référence), que ces éléments soient des personnages, des (séquences d') événements ou des mondes fictifs; quant aux "textes", il peut s'agir aussi bien de textes au sens strict (romans, nouvelles, mais aussi essais dans certains cas) que de films, bandes dessinées, épisodes télé, etc. La notion recouvre des pratiques aussi diverses que la reprise de personnages telle qu'on l'observe dans la Comédie humaine, les suites (autographes ou allographes), les séries, la retraversée d'une diégèse dans une perspective différente, la modification d'une intrigue antérieure (comme dans Emma, oh! Emma! de Cellard, où Emma Bovary ne se suicide pas), la réunion de personnages appartenant à des mondes fictifs distincts (Sherlock Holmes vs. Dracula de Loren Estleman) et quelques autres formules encore. (Saint-Gélais, 2012).

Une fiction est transfictionnelle lorsque son référent est une autre fiction, comme c'est le cas de Petits Chaperons. En effet, comme je l'ai déjà signalé, le référent des micronouvelles n'est ni le texte de Perrault ni le texte de Grimm mais la fiction dont ces deux textes sont des versions. Le Petit Chaperon Rouge est un bel exemple de l'autonomie de la fiction par rapport aux textes qui la configurent dans des récits spécifiques. De cette autonomie le personnage est un opérateur clé, vu sa capacité de transposer les frontières des textes et des récits. La quatrième de couverture affirme que Zárate écrit des micronouvelles autour de contes et de personnages célèbres. Le Petit Chaperon Rouge participe des deux catégories. C'est effectivement un personnage mémorable qui, comme les autres personnages des contes - Cendrillon, Blancheneige, Petit Poucet, etc. - s'est détaché et émancipé du récit qui l'a instauré, celui de Perrault, pour devenir le protagoniste d'un grand nombre d'autres récits matérialisés en des arts et des supports différents, depuis le conte de Grimm jusqu'à Red Riding Hood, un film tourné par Catherine Hardwick en 2011. Le Petit Chaperon Rouge est ce que Richard Saint-Gélais appelle un personnage migrant dont la mouvance signale le décollage du monde fictionnel, qu'il polarise et représente, par rapport aux récits qui lui donnent forme et expression et qui en constituent autant de versions. C'est un marqueur de transfictionnalité. Définir le personnage comme marqueur transfictionnel implique qu'il agit dans l'histoire et non pas qu'il est seulement cité, évoqué ou référé. II faut distinguer entre une allusion à Cendrillon en tant que modèle du Petit Chaperon: "Douze coups de minuit. Le chemin long devint court. Le Petit Chaperon une cendrillon, et le loup féroce un prince sans imagination au lit." (Zárate, 2011:48); et un récit où Cendrillon joue un rôle actantiel comme dans cette microfiction de Ana Maria Shua: "A la doce en punto pierde en la escalinata del palacio su zapatito de cristal. Pasa la noche en inquieta duermevela y retoma por la mañana sus fatigosos quehaceres mientras espera a 
los enviados reales. (Princípe fetichista, espera vana.) “ (Shua, 2007:70). Dans le premier cas il s'agit d'un lien intertextuel tandis que le second est un lien transfictionnel.

\section{Figures transfictionnelles}

Parler de transfictionnalité revient donc, pour l'analyse, à quitter le niveau de la structure ou forme narrative (la matérialité discursive) pour celui du contenu de l'histoire (le matériau diégétique). Car c'est aussi sur ce plan que les micronouvelles de Zárate déstabilisent le conte en mettant en place des incompatibilités diégétiques. Chaque petit récit retraverse un segment de l'histoire pour en donner une vision différente ou, ce qui est plus fréquent, altère les données diégétiques établies au moyen d'additions contrefictionnelles, c'est-à-dire l'introduction d'éléments étrangers à la fiction standard: actions, personnages, objets.

La micronouvelle 75 revisite le segment diégétique compris entre la dévoration de la Grand-Mère par le Loup et la mort de celui-ci par le Chasseur: "Le loup était habillé en mèregrand, mais la mort arriva habillée en chasseur'. Le récit condense la séquence, qui aboutit à la dévoration de la fillette, tout en éliminant le dialogue au lit entre elle et le Loup déguisé en Grand-mère. Mais la condensation n'altère pas la trame. La micronouvelle 68 réécrit l'histoire sous une perspective différente: "J'ai été le Petit Chaperon rouge, se dit-elle, en touchant avec nostalgie le tissu, le panier plein de poussière, la hache oxydée, le toujours fidèle tapis en peau de loup'. Ce monologue de la protagoniste retraverse les nœuds privilégiés du conte en énumérant ses objets-fétiches - chaperon, panier, hache, dépouille du loup - sous un angle subjectif qui infléchit rétroactivement le profil du personnage: non pas une fillette innocente, mais plutôt une ex-star nostalgique de son passé.

L'opération transfictionnelle la plus employée est la contrefiction: "Le loup n'arriva jamais chez la mère-grand. Le Petit Chaperon s'y prenait très mal pour indiquer les directions" (61). Ce récit est entièrement contrefictionnel, car il altère de fond en comble la trame officielle, en posant ce qui serait arrivé si, à tel point, I'histoire avait pris un sens différent: au lieu de donner des informations précises au Loup, le Petit Chaperon l'a désorienté. Dans la micronouvelle 4, citée plus haut, l'ajout du syntagme "depuis longtemps morte" suffit à altérer les données diégétiques. Le récit pose le Petit Chaperon sans GrandMère. La Grand-Mère a été retirée avec sa petite-fille du ventre du Loup, chez Grimm; ou alors elle est bel et bien morte avec sa petite-fille, chez Perrault. Mais il n'y a pas de Petit Chaperon sans Grand-Mère, sauf dans cette version de Zárate qui prolonge l'histoire au-delà de la mort de l'aïeule. La micronouvelle 17, déjà citée aussi, procède pareillement: c'est la subordonnée temporelle qui introduit la donnée contrefictionnelle dans une version qui semblait jusque-là suivre la trame établie: au lieu d'avoir tué le Loup, le Chasseur a tué le 
Petit Chaperon accusé de libertinage. La micronouvelle 57 donne une version différente de la mort du Petit Chaperon, cette fois-ci victime de meurtre collectif. Ces dénouements coexistent avec ceux où la protagoniste a survécu (est re-née). C'est le cas du texte 37 qui modifie un petit détail de la fiction officielle. Celle-ci dit que le Chasseur a pris la peau du Loup et l'a emportée chez lui. Dans la microfiction il l'a donnée au Petit Chaperon: "Combien est délicat le pelage du loup, combien il est doux sur le corps nu, combien il est aimable le chasseur, de lui avoir apporté cette peau" (37). Entre douceur de l'un et amabilité de l'autre, le texte suggère un portrait en femme fatale, capricieuse, sensuelle et meurtrière. La diversité de dénouements logiquement incompatibles assigne au personnage des destins différents et c'est pourquoi il n'y a pas un mais des petits chaperons. Mais quoi qu'il en soit de la diversité des déroulements, des dénouements et des destins de l'héroïne, celle-ci garde un seul et même profil, celui de fausse innocente (à la différence des autres personnages ainsi que du Petit Chaperon de Lascault). Ce profil cohérent hypostasie le personnage et contribue à l'unité sémantique des micronouvelles. Le conte porte sur une fillette naïve qui sort de l'enfance. L'expérience traumatique qu'elle vit avec le Loup la libère de sa naïveté et la prépare à une nouvelle étape de la vie, la puberté, qui implique "la perte de l'innocence" - expression stéréotypée dont la portée signifiante se déploie entre perte de l'insouciance infantile et perte de la virginité. Dans la série microfictionnelle, la fillette est depuis longtemps sortie de l'enfance, a depuis longtemps perdu l'innocence qui n'est qu'un masque: "Le Petit Chaperon s'approche du lit. Le déguisement de mère-grand est moins crédible que son déguisement d'innocente" (70). Ce qui dans le conte est une fillette victime de séduction, devient dans les micronouvelles une séductrice ${ }^{15}$. Est-ce bien le même Petit Chaperon Rouge? La contrefiction est une forme limite de transfictionnalité dans la mesure où elle exacerbe les tensions entre identité et altérité (Saint-Gélais, 2011:163). Le Petit Chaperon de Zárate est le même et l'autre que celui du conte: le même, car pétri dans la pâte thématique de la séduction; l'autre, car sa passivité devient activité. C'est le Petit Chaperon possible dans un monde cynique et ludique. Ceci illustre une idée de Dolezel, selon laquelle les réécritures postmodernes se fondent dans la contingence des mondes possibles: "chaque monde et chaque entité dans le monde auraient pu être différents de ce qu'ils sont" (Dolezel, 1998: 222).

La contrefiction emprunte aussi la voie de tirer le monde fictionnel (l'hypotexte) vers le temps de la production du récit transfictionnel. Le "il était une fois" du conte s'aplatit dans la banalité de notre vie quotidienne. II ne s'agit pas de transplanter les personnages dans un milieu urbain contemporain mais d'introduire dans le temps jadis du conte certains éléments,

\footnotetext{
${ }^{15}$ Voir la micronouvelle 8, citée dans la note 11, où le Petit Chaperon, loin d'être la proie des loups, tourne à son avantage la stratégie maternelle
} 
technologiques et autres, qui renvoient immédiatement à notre contemporanéité (Facebook, chats, youtube, GPS). La contrefiction assume les changements diégétiques causés par la présence de ses éléments: quel tournant aurait pris l'histoire si le Petit Chaperon disposait d'un GPS? La forêt s'urbanise en lieu de rencontres sexuelles mais elle devient surtout un espace hétéroclite où co-existent internet, moutons, embouteillages, chasseurs, psychiatres, touristes, paniers, pizzas.

Le croisement est une autre figure transfictionnelle qui opère une conjonction de deux fictions indépendantes. C'est un peu comme faire un puzzle avec des pièces de puzzles différents. Principe structurant du crossover, genre foisonnant dans la culture populaire et médiatique, le croisement, qui peut être autographe ou allographe, est un dispositif ludique. Quatre micronouvelles croisent le Petit Chaperon et les trois petits cochons ${ }^{16}$, une rencontre favorisée par le protagonisme du Loup dans les deux contes: "On disait que sa jalousie était déplacée, mais le Petit Chaperon ne pouvait s'empêcher d'éprouver de la haine envers les trois petits cochons." (6); "Le chasseur sortit du loup, miraculeusement encore en vie, le Petit Chaperon, la mère-grand, trois petits cochons et un homme perdu qui dit s'appeler Geppetto" (23). "Ils ouvrirent, en toute innocence: qu'avaient à craindre les trois petits cochons du Petit Chaperon?" (33). "Après avoir rendu visite au Petit Chaperon, le loup ne peut abattre d'un souffle la maison de paille du petit cochon." (34). L'introduction de ces personnages issus d'un autre conte met en place un triangle amoureux impliquant le Petit Chaperon, le Loup et les Trois Petits Cochons, fondé dans le sémantisme sexuel de la dévoration. Dans la micronouvelle 33, le personnage qui frappe à la porte peut aussi bien être le Loup déguisé en Petit Chaperon que le Petit Chaperon lui-même qui, en pleine crise de jalousie, vient tirer satisfaction de l'injure ou alors, pourquoi pas, désire élargir son champ d'expériences sexuelles (on ne sait pas de quoi les trois petits cochons ne se méfient pas: de sa jalousie ou de sa libido). La micronouvelle 23 ajoute à la réunion de mondes fictionnels celui de Pinocchio dont le père a été avalé par le Loup. II n'y a pourtant pas de loup dans I'histoire de Pinocchio (qui se fait avaler par une baleine), ce qui approfondit l'étrangeté de ce croisement et justifie l'égarement de Geppetto.

Le croisement ne se borne pas au champ des contes pour enfants. La première micronouvelle transporte le Petit Chaperon et le Loup dans le monde biblique de Noé (l'arche, couple de chaque animal), le re-fondateur postdiluvien de la biodiversité du monde animal: "Un couple de chaque animal, mais le Loup refuse de monter dans l'arche sans le Petit Chaperon." (1). Que ce texte soit le premier du recueil n'est certainement pas dû au hasard, la visée refondatrice du programme de Noé patronnant la refondation d'un monde de

\footnotetext{
${ }^{16}$ Le conte des Trois Petits Cochons ne fait pas partie du canon Perrault-Grimm. The Big Bad Wolf (1934), dessin animé de Disney, réunit également le Petit Chaperon et les trois petits cochons.
} 
fiction. Mais là où la Bible raconte que Noé procède à une discrimination des espèces, chacune étant représentée par un couple en vue de leur reproduction en ordre, les microfictions de Zárate racontent la promiscuité interspecies. Aussi les deux personnages se trouvent-ils déplacés dans le projet de préservation des espèces, le terme "déplacés" ayant le double sens de passage ou transférence (déplacés vers) et de mal intégrés, étrangers, inadaptés, sans place, pour autant qu'ils forment un couple d'espèces différentes (déplacés dans).

Ces trois opérations transfictionnelles - la retraversée, la contrefiction et le croisement - s'ordonnent selon la portée croissante de la révision infligée au matériau diégétique de référence. Étant donnée la discontinuité parataxique des récits, il y a autant de versions du conte que de micronouvelles, chaque version étant plus ou moins éloignée, plus ou moins transgressive, plus ou moins déformante ou disruptive, selon la figure transfictionnelle adoptée. Mais il ne semble pas hors de propos de considérer la série comme une version du conte, une version certes polymorphe, hétérogène et détotalisée, mais dont la cohésion est assurée par l'unité thématique des amours antispécistes. Les micronouvelles partagent un sol sémantique commun caractérisé par l'hyper-érotisation des rapports entre les personnages. On trouve dans la série de Lascault quelques textes sur les amours du Petit Chaperon et du Loup - "Le loup a posé sa tête sur les genoux du Petit Chaperon Rouge. Elle lui gratte doucement le crâne. II neige au bord du lac" - mais tous les récits ne traitent pas ce sujet. Chez Zárate, par contre, ces amours antispécistes constituent le seul thème de la série et engagent non seulement le couple protagoniste mais aussi le Chasseur et la Grand-mère dans un réseau de partenaires échangeables - "Innocence ou connaissance? Découverte ou expérience? Petit Chaperon ou mère-grand?" (21) -, aux identités fluides - "Le Loup s'habillait en mère-grand, le chasseur en Petit Chaperon. Ce bois était vraiment mal fréquenté." (27); "Le Petit Chaperon était l'appât. La mère grand mangeait souvent du loup" (50).

L'isotopie $^{17}$ sexuelle oriente la démarche réinterprétative à l'œuvre dans la transfictionnalité. En effet, ses opérations interfèrent rétroactivement sur la fiction standard avec plus ou moins de vigueur, selon qu'elles proposent un nouvel aperçu de l'histoire ou d'une de ses séquences ou qu'elles en altèrent le déroulement et/ou les faits, et entraînent implicitement une réinterprétation de l'œuvre, en l'occurrence le conte. En tant que version alternative traversée par l'isotopie sexuelle, Petits Chaperons déniaise l'objectif éducatif du conte, en exploitant le sémantisme sexuel dont l'histoire est imprégnée. II est clair que le loup du conte n'est pas un loup mais, à la façon des fables, un homme et que sa faim est

\footnotetext{
${ }^{17}$ L'isotopie consiste dans l'itérativité de classèmes (sèmes contextuels récurrents) qui assurent à l'énoncé son homogénéité (Greimas, 1993:197).
} 
métaphorique. La couleur rouge du chaperon met en évidence la beauté pubère de la fillette et suggère son éveil à la sexualité ${ }^{18}$. C'est un conte pseudo-naïf qui met en garde contre les dangers que courent les jeunes filles désinformées (Perrault) ou désobéissantes (Grimm). Zárate déniaise cette mise en garde, en explicitant, c'est-à-dire en "dépliant" le sémantisme sexuel plié (sous-entendu) dans les métaphores: "Perrault regarde l'illustration de son conte. Certains pourraient faire une remarque sur le dessin du Petit Chaperon entrant dans le lit avec le loup. Ou le fait que ce dessin soit dépliable." (67). La dernière phrase est elle-même un pli métaphorique qui mi-dit pudiquement ce que cache le dessin soi-disant naïf. Les micronouvelles déplient les sous-entendus du conte et en dévoilent la signification érotique. Le loup n'est pas une allégorie, c'est un loup, certes pourvu de qualités humaines, mais un loup qui s'engage dans une relation érotique avec une fille qui fait semblant d'être naïve. Ce qui corrige le dénouement tragique de la version de Perrault n'est pas l'extension en happy end de la version de Grimm, mais la version hypersexualisée de Zárate. Cette correction est mise au compte du personnage: "-Perrault affirme qu'au final, ils trouvèrent la mort. Le Petit Chaperon sourit. - Juste la petite." (46). Ce n'est pas un conte pour enfants, c'est un conte pour adultes, et la moralité est remplacée par la dérision: dérision du conte, de son but éducatif et de la validité de son dénouement; mais aussi autodérision dans la neutralisation réciproque de la validité des dénouements multiples et incompatibles.

Le dispositif transfictionnel de Petits Chaperons compte une quatrième figure, celle que Saint-Gélais appelle la capture, consistant à adjoindre à un texte "un autre qui l'enchâsse tout en lui donnant une manière de prolongement diégétique (2011:251). II s'agit d'un procédé métaleptique qui télescope le dedans et le dehors de la fiction: la diégèse originale devient un récit, un livre, dans la diégèse transfictionnelle, comme dans le second volume de Don Quijote où le premier volume est un livre dans le livre. L'effet vertigineux du court-circuit entre réalité et fiction tient à la capture du réel dans la représentation, si bien que l'œuvre dont les personnages ont été tirés est lue par eux: "Le Petit Chaperon échappe au loup. Elle parvient à fermer, au dernier moment, le livre de Perrault" (69). II en va de même de l'auteur. Chez Zárate, Perrault est capturé dans la fiction qui englobe la fiction qu'il

\footnotetext{
18 La reconnaissance et l'explicitation du sémantisme sexuel des contes de fées constitue une tradition herméneutique dont la psychanalyse est un des agents majeurs. Les psychanalystes post-freudiens et les anthropologues de ligne freudienne qui se sont penchés sur les contes de fées, comme Bruno Bettelheim et Nicole Belmont, les définissent comme des mises en récit d'opérations psychiques fondamentales du développement libidinal de l'enfant, au cours duquel l'entrée dans la puberté et l'accès à la sexualité sont des moments spécialement problématiques (Bettelheim, 1976:13-36, Belmont, 1999:154, 211). Dans le cas du Petit Chaperon Rouge I'histoire est lue comme une figuration d'une phase du processus de maturation psychosexuelle de la fillette: le rouge symbolise l'éveil sexuel dont le sang menstruel est le signe bio-physiologique, le Grand Méchant Loup incarne les dangers de la séduction et l'angoisse du sexe, la séquence au lit figure la scène primitive dans laquelle l'acte sexuel est perçu comme agression, la jouissance fantasmée comme dévoration. La visée éducative du conte se renforce en s'annexant un effet thérapeutique: le subtil dispositif figuratif de la fiction, en drainant l'inconscient en imagination, aide les enfants à surmonter les angoisses et les impasses du processus de maturation psychosexuelle et finalement à sortir de l'enfance (ne plus voir dans l'autre sexe quelque chose de menaçant).
} 
a écrit; il est absorbé dans la fiction qui dérive de son conte. C'est ce qui se passe dans la micronouvelle 67, déjà citée, où Perrault regarde le dessin dépliable qui illustre son conte. Dans le texte 63, il intervient auprès du personnage, comme le ferait un metteur-en-scène, pour assurer le déroulement de l'action: "Elle s'amusa tellement sur le chemin long que Perrault dut aller la voir pour qu'elle continue le conte." (63). Dans la micronouvelle 46, citée plus haut, c'est le personnage qui corrige l'auteur, en remplaçant le dénouement heureux (érotique) au dénouement malheureux. Le témoignage du Petit Chaperon semble plus fiable que celui de l'auteur puisqu'elle a vécu les événements alors que lui n'a fait que les écrire: la fiction enchâssante se donne ironiquement pour plus réelle que la fiction originale. En tant que personnage, Perrault a un statut à part. II n'a pas affaire aux autres personnages au sein du réseau érotique, il a affaire au conte, à sa production et à sa réception: "Si je coupe tout ça, dit Perrault au censeur, il ne restera qu'un conte pour enfants." (49); "Entre les lignes: viol, mort. Un bon conte pour enfants. Ils encensent Perrault. Ils sont d'accord avec l'idée selon laquelle la terreur est pédagogique." (80). Ces micronouvelles ont une fonction critique et métadiscursive par laquelle l'œuvre participe au vieux débat sur le destinataire des contes. Auteur devenu personnage, Perrault désigne le lien complexe entre la micronouvelle et le conte: rapport de dérivation et de réappropriation du conte, à travers le travail de déconstruction ludique de sa forme, de désintégration de sa narrativité, de recyclage ironique et parodique de son imaginaire; bref un travail de détotalisation sérielle effectué sur la structure, la forme et le contenu du conte, qui contribue puissamment à préserver sa mémoire dans un sens non pas rigide et muséal mais dynamique et revitalisant.

\section{Microrécits, mondes immenses}

Nous voyons que l'action de la série microfictionnelle sur le conte est à double tranchant. Sur le plan du discours narratif, elle désintègre le conte en une pluralité de petits récits qui ne s'articulent pas mais s'accumulent (rupture de l'axe de la syntagmatique narrative). Par contre, sur le plan du contenu, la série procure au flux des micronouvelles une certaine cohésion et homogénéité grâce au monde de fiction du Petit Chaperon rouge et à l'isotopie sexuelle. Les micronouvelles sont des versions alternatives de la même fiction qui suivent la même thématique, ce qui détermine la série comme version alternative emboîtante. L'unité des micronouvelles s'établit sur l'axe paradigmatique (fiction de référence, lexique sexuel). Mais il y a un autre aspect où la série opère une mise en ordre. $\mathrm{Si}$ la mise en série des microfictions - ou, selon le mot de Lauro Zavala "la naturaleza gregaria del género" - est de règle, c'est parce que la série contribue à réguler le rapport inversement proportionnel entre quantité textuelle minimale (dimension du texte) et amplitude maximale du monde induit (dimension du monde). En se bornant à mentionner l'état de choses de son 
monde de fiction (absence de descriptions, explications, caractérisations), une micronouvelle produit une "texture zéro" dans laquelle le non-dit, le non-narré ou dé-narré pèse bien davantage que le narré. Tout monde fictionnel est incomplet et comporte des zones indéterminées (qui était le père du Petit Chaperon?) mais la micronouvelle fait du principe d'incomplétude son propre mode de structuration et de fonctionnement (Macé, 2010: 217). Les mondes ainsi créés ou recréés présentent un degré d'accessibilité très bas qui empêche l'immersion empathique du lecteur dans la fiction et l'oblige, au contraire, à dépenser un grand effort herméneutique. C'est l'effet que produit le Dinosaurio, de Augusto Monterroso: "Cuando despertó, el dinosaurio todavía estaba alli". Ce microrrelato, célèbre parmi tous, invite le lecteur à remplir le dé-narré, à peupler et à meubler le vaste désert qui entoure le dinosaure. Par son effet de répétition (de traits stylistiques, de conventions génologiques, de lexèmes, d'entités fictionnelles), la série fournit un principe de cohésion qui réduit le degré d'indétermination du monde, en lui donnant un contour qui permet au lecteur de se repérer tant soit peu. La série des nouvelles en trois lignes de Fénéon, par exemple, répète, en les altérant, les conventions de forme et de contenu des faits divers. Cette marque génologique, qui définit les nouvelles en trois lignes comme des réécritures de faits divers, constitue le principe de cohésion de la série et oriente la lecture. La transfictionnalité renforce cet effet dans la mesure où la référence à une fiction pré-existante réduit considérablement l'amplitude du monde. À plus forte raison lorsque la fiction pré-existante est un conte de grande circulation et de grande consommation. Dans son autonomie, la micronouvelle suivante produit un monde d'une immense étendue: "Soupirant, sussurrant, elle m'aime un peu - beaucoup - passionnément - à la folie - pas du tout, le loup effeuillait des moutons" (29). L'ironie est immédiatement perceptible dans le contraste entre le jeu romantique d" effeuiller la marguerite et la substitution des pétales par des moutons. Mais on ne sait plus rien de ce vaste monde où un loup amoureux avale des moutons. Une fois cette micronouvelle insérée dans la série de Petits Chaperons, l'étendue du monde devient celle de la fiction de référence et on comprend que "elle", c'est le Petit Chaperon, et que cette micronouvelle est une version contrefictionnelle thématisant les amours du couple antispéciste. La série a un effet de stabilisation du monde et du sens qui contrecarre l'instabilité produite par les figures ludiques et corrosives de la transfictionnalité (contrefiction, croisement) dans chaque petit récit. Bref, une micronouvelle dé-narre, la série pro-narre.

\section{Conclusion}

L'étude de Petits Chaperons de José Luis Zárate nous a permis de situer la connexion de la microfiction au conte à deux niveaux d'analyse. Au niveau formel, le rapport intertextuel à un conte particulier se configure comme une métamorphose du petit tout en 
des tout petits. En brisant la cohérence syntagmatique du conte, l'organisation parataxique les micronouvelles fait éclater l'unité narrative qui caractérise le conte, ce qui affecte spécialement le temps, aussi bien à l'intérieur de chaque micronouvelle (temps reployé) qu'à l'extérieur (temps désorienté). Mais il y a des forces centrifuges et des forces centripètes à l'œuvre dans cette mutation de la forme narrative. Là où les forces centrifuges ont désintégré l'axe horizontal des séquences, les forces centripètes travaillent à établir entre les petits récits flottants une cohésion verticale de nature paradigmatique (lexique des personnages, isotopie). C'est ce que le concept de transfictionnalité nous permet d'articuler. La métamorphose est maintenant d'ordre substantiel, car les opérations transfictionnelles affectent directement le contenu diégétique et sémantique du conte auquel elles infligent des transformations et des déformations. Mais cette continuité verticale ne récupère aucunement la continuité horizontale du temps. Le temps en tant qu'il se déploie en temporalité semble être la catégorie du récit la plus affectée dans les micronouvelles, genre qui mise sur la fugacité de l'instant. Le principe même d'organisation paradigmatique de la série met en relief la discontinuité des multiples versions alternatives - autonomes malgré l'isotopie - de la même fiction. La série fait ainsi ressortir la discontinuité comme trait majeur de la mutation du conte en microfiction. Dans le cadre du débat autour des formes et des régimes de cette mutation, Petits Chaperons pointe vers une légitimation de la micronouvelle (ou microrécit ou microfiction) comme genre autonome, dérivé du conte certes mais, comme le dit Lagmanovitch, par rupture.

\section{Bibliographie}

ANDRES-SUAREZ, Irene (2010). El microrrelato español. Una estética de la elipsis. Palencia: Menoscuarto.

BAudelaiRe, Charles (1965) "Notes nouvelles sur Edgar Poe". In: POE, Edgar Allan. Nouvelles histoires extraordinaires. Paris: Flammarion.

BELMON, Nicole (1986) Paroles païennes. Mythe et folklore. Paris: Imago. (1999). Poétique du conte. Essai sur le conte de tradition orale. Paris: Gallimard. BENJAMIN, Walter (1991). Écris français. Paris: Gallimard.

Bettelheim, Bruno (1976). Psychanalyse des contes fées. Paris: Laffont.

BONNET, Gilles (2011). "Macrocities/microstories: A Brave New Digital World (L'Écranvain et la nouvelle-écran: pour une poétique de la fiction numérique'. In: Cristina Álvares et Eduarda Keating (orgs.), (2011). Atas do Simpósio Internacional "Microcontos e outras microformas". Braga: CEHUM;pp. 4-9. 
FARIA, Dominique (2009). "Lire un blog: L'autofictif d'Éric Chévillard”. In: Carnets. Cultures littéraires: nouvelles performances et développement, numéro spécial automne/hiver, pp.173-181.

FÉNÉON, Félix (1998) Nouvelles em trois lignes. Paris:LGF.

GREIMAS, Algirdas Julien et COURTES, Joseph (1993). Sémiotique. Dictionnaire raisonné de la théorie du langage. Paris: Hachette.

HeIDMANN, Ute et AdAM, Jean-Michel (2010). Textualité et intertextualité des contes. Perrault, Apulée, La Fontaine, Lhéritier. Paris: CNRS.

LAGMANOVITCH, David (2006). El microrrelato. Teoría e historia. Palencia: Menoscuart.

LAWRENCE, James Cooper (1917). "A Theory of the Short Story". In: The North American Review, Vol. 205, No. 735, pp. 274-286.

LYOTARD, Jean-François (1979). La condition postmoderne. Paris: Minuit.

MACÉ, Marielle (2010) “'Le total fabuleux': les mondes possibles au profit du lecteur”. In: Lavocat Françoise, org. (2010). La théorie littéraire des mondes possibles. Paris: CNRS, pp.205-222.

NUÑEZ-SABARíS, Joaquín (2012) "Génesis y consolidación del microrrelato español" (texto comunicado pessoalmente, a publicar).

PAVEL, Thomas (1988). Univers de la fiction. Paris: Seuil.

PINKOLA ESTES, Clarissa (1996). Femmes qui courent avec des loups. Paris: Grasset (1992).

QUIGNARD, Pascal (2005). Une gêne technique à l'égard des fragments. Paris: Galilée (1984).

ROAS, David, dir. (2010). Poéticas del microrrelato. Madrid: Arco Libros.

SAINT-GÉLAIS, Richard (2011). Fictions transfuges. Paris: Seuil. (2012), "Fictions transfuges.La transfictionnalité et ses enjeux. Entretien. Propos recueillis par Frank Wagner" [en ligne]. In: Vox poetica [disponible en avril 2013] <URL: http://www.vox-poetica.org/entretiens/intStGelais.html>.

SHuA, Ana Maria (2007) Casa de geishas. Barcelona: Thule.

TREVISAN, Donald (2003). O vampiro de Curitiba. Rio de Janeiro: Record.

VALLS, Fernando (2008). Soplando vidrio y otros estudios sobre el microrrelato español. Madrid: Páginas de Espuma.

ZARATE, José Luis (2011) Petits Chaperons. Paris: Outworld.

ZAVALA, Lauro (1999) "Seis problemas para la minificción, un género del tercer milenio: Brevedad, Diversidad, Complicidad, Fractalidad, Fugacidad, Virtualidad" [en ligne]. In: Anuario de investigación 1998, Vol.1, México DF: UAM-X [disponible en avril 2013] <URL: http://www.ciudadseva.com/textos/teoria/hist/zavala2.htm>. (2002). "El cuento ultracorto bajo el microscopio". In: Revista de literatura, 64, 128, pp.539-53. 
(2004) "La teoría del cuento y la minificción en Venezuela". In: Anuario de investigación 2003, Vol.6, México DF: UAM-X, pp.126-34.

(2009) "Los estudios sobre minificción: Una teoría literaria en lengua española".

In: El cuente en red. Revista eletrónica de teoría de la ficción breve, 19, pp. 37-44.

(s/d) "Para analisar a minificção", Minguante <URL:

http://www.minguante.com/intro.asp> (ce site n'existe plus). 